EPJ Web of Conferences 41, 11002 (2013)

DOI: $10.1051 /$ epjconf/20134111002

(C) Owned by the authors, published by EDP Sciences, 2013

\title{
Programmable broadband ultra-fine resolution 2-D pulse shaping
}

\author{
A. J. Metcalf ${ }^{1}$, V. Torres-Company ${ }^{1,2}$, V. R. Supradeepa ${ }^{1,3}$, D. E. Leaird ${ }^{1}$ and A. M. Weiner ${ }^{1}$ \\ ${ }^{1}$ School of Electrical and Computer Engineering, Purdue University, West Lafayette, IN 47906, USA \\ ${ }^{2}$ Department of Physics, Universitat Jaume I, 12071 Castello de la Plana, Spain \\ ${ }^{3}$ Current address: OFS research laboratories, New Jersey, USA
}

\begin{abstract}
We demonstrate a fully programmable two dimensional pulse shaper, capable of fine resolution control over a broad bandwidth. Experimental results show line-by-line shaping of frequency combs, highlighting an application in radio frequency arbitrary waveform generation.
\end{abstract}

\section{Introduction}

Fourier pulse shaping is a widely adopted technique used to manipulate the spectrum of short pulses to achieve user-specified optical waveforms [1]. An important aspect in many applications is the ability to control fine spectral features over a broad bandwidth allowing for a high time-bandwidth product (TBWP). However, in traditional 1-D shapers there is a fundamental trade-off between the two. We overcome this limitation by using a shaping apparatus that disperses light into two dimensions, taking advantage of both a virtually imaged phased array (VIPA) and a grating as spectral dispersers [2]. Basic programmability, but with manual control, was achieved in [3] by introducing a two-dimensional liquid crystal on silicon (LCOS) spatial light modulator (SLM), a device consisting of $\sim 2$ million pixels. Here we demonstrate full programmability with automatic control, with capability of controlling $\sim 3 \mathrm{GHz}$ features over $5 \mathrm{THz}$ bandwidth, leading to potential for TBWPs $>1500$. Experimental results are presented, including operation in a closed-loop configuration, as well as an application in radio frequency arbitrary waveform generation (RF-AWG).

\section{Experimental setup and results}

The setup is shown in Figure 1. The incident light travels through the VIPA where it is dispersed in the vertical direction. Due to the degeneracy in the VIPA, wavelengths which are separated by integer numbers of the VIPA FSR $(200 \mathrm{GHz})$ overlap spatially. Next the light is sent through a transmission grating which breaks up the spatial degeneracy of the VIPA by dispersing light horizontally before being focused onto the SLM. The mask is formed by grouping pixels into 'Superpixels' consisting of 10x10 pixels, which can positioned with an addressability of $\sim 600 \mathrm{MHz}$. Amplitude shaping is accomplished by controlling the phase excursion of a finely spaced sawtooth grating within the superpixel, whereas phase shaping is achieved by altering the average phase offset [4]. 
a)

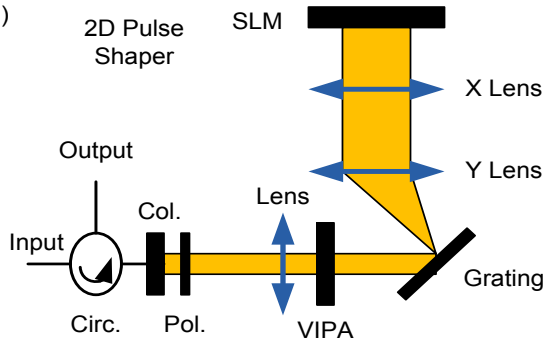

c)

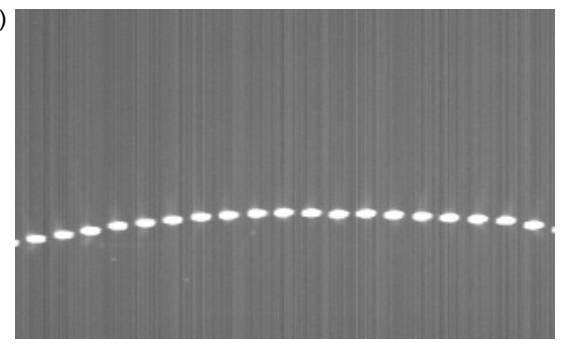

b)

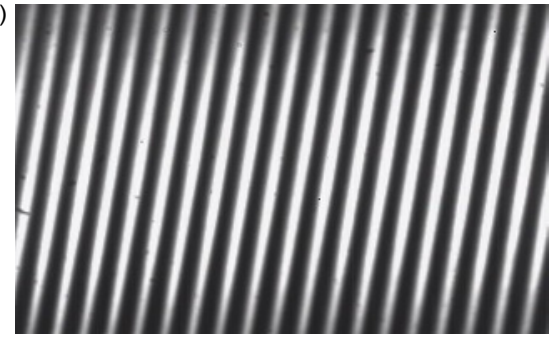

d)

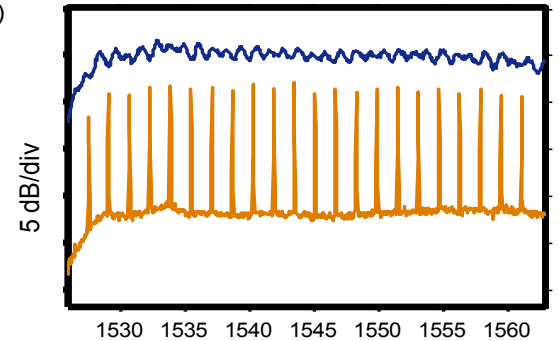

Fig. 1. (a) Schematic representation of the 2-D shaper setup, (b) image of Fourier plane, (c) image after mask is applied, one feature every $1.6 \mathrm{~nm}$, (d) spectrum (nm) corresponding to b) and c).

The fine resolution capability of this shaper makes it ideal for line-by-line shaping of optical frequency combs [5]. In our first experiment, we demonstrate control of a frequency comb generated by passing a CW laser through 2 intensity modulators and a phase modulator driven at $5 \mathrm{GHz}$, leading to 40 lines spaced by $5 \mathrm{GHz}$ within $10 \mathrm{~dB}$ bandwidth. The inherent spectral phase of the comb was compensated with $1.4 \mathrm{~km}$ of SMF fiber before being spectrally broadened using a dispersion-decreasing-fiber soliton compressor [5]; resulting in 250 lines within $15 \mathrm{~dB}$ [see Figure 2(a)] and a pulse duration of $\sim 2 \mathrm{ps}$ full width at half maximum. The pulse train is then measured using a $22 \mathrm{GHz}$ fast photodiode and monitored using a sampling oscilloscope [see Figure 2(b)]. To show simultaneous amplitude and phase control we double the repetition rate by applying an amplitude mask that kills off every other line, a linear spectral phase is then applied, shifting the synthesized waveform in time [see Figure 2(c)]. To demonstrate the full capability of the device we operate it in a closed loop setup where the sampling scope acts as a feedback device. Here we use a phase only mask that takes advantage of the Talbot effect to multiply the pulse train to $15 \mathrm{GHz}$. The program then monitors the waveform while varying the imposed phase values for optimization. In this example the intensity variations improved from $\sim 16 \%$ down to $\sim 3 \%$ in only 8 iterations [see Figure 2(d)].
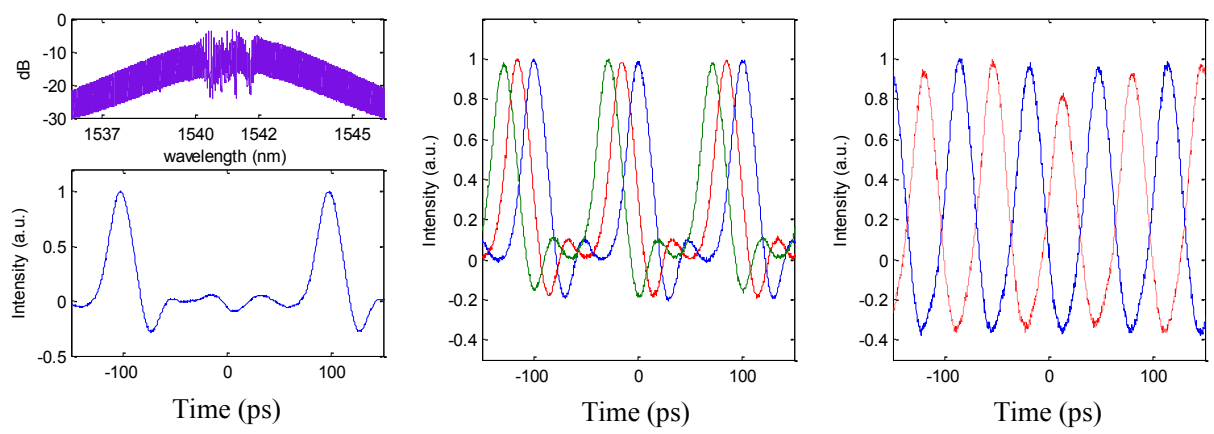

Fig. 2. (a) Input spectrum of $5 \mathrm{GHz} F C$ (b) Original 200ps pulse train (c) 100 ps pulse train shifted by 15 and 30ps, respectively (d) $15 \mathrm{GHz}$ tone after 3x Talbot mask applied, dashed: before learning loop, solid: after learning loop. 
In the next example we focus on a method to rapidly switch between waveforms in photonically assisted RF-AWG. In RF-AWG we desire line-by-line shaping in order to achieve waveforms that span the full time aperture, as well as the ability to rapidly switch between them. However, switching times are limited on the order of milliseconds due to SLM refresh rates. Here we use a switching scheme presented in [6], allowing switching within a clock cycle (200ps). The switching mechanism rapidly chooses between four separate $5 \mathrm{GHz}$ combs with different center frequencies. The shaper is programmed with one static mask that incorporates a different waveform for each comb. It is then up to the switching mechanism to pick what comb is incident on the shaper to determine which mask is illuminated.
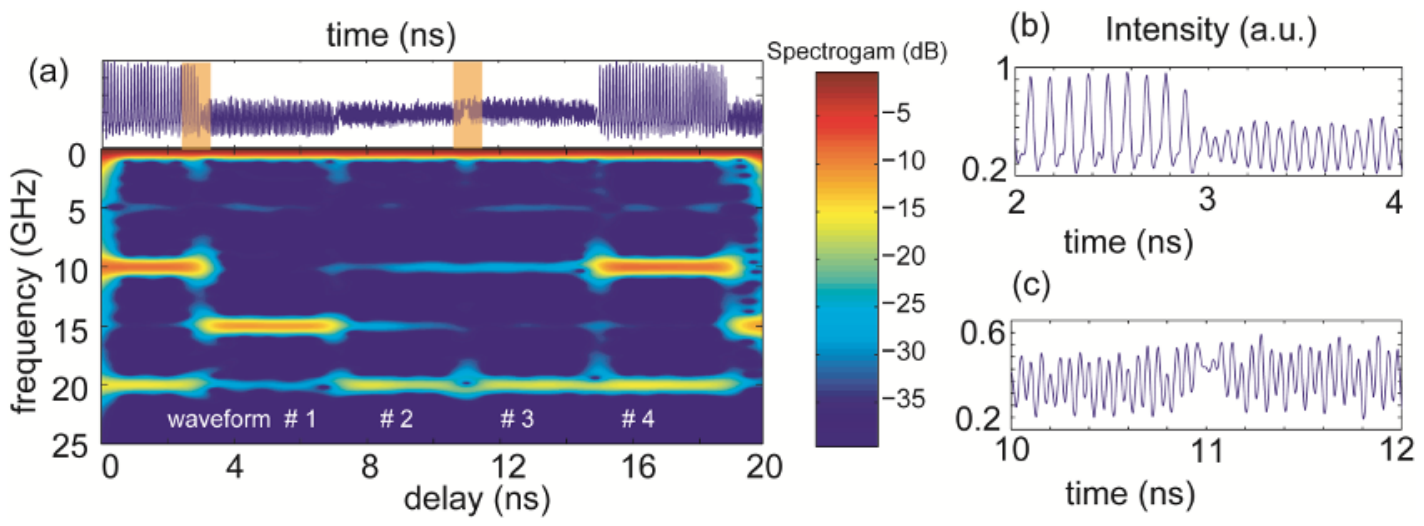

Fig. 3. (a) Single-shot intensity snapshot of the synthesized sequence (top) and calculated spectrogram (bottom) consisting of 4 different waveforms. (b) and (c) zoom-in parts corresponding to the shadow regions in (a).

The masks were all created by employing the temporal Talbot effect. The rapidly switched waveforms were recorded using a real-time scope, with a typical sequence of $4 \mathrm{~ns}$ per waveform shown in Figure 3(a), together with the computed spectrogram. Figures 3(b) and (c) are a zoom-in of transitions between waveforms \#4-\#1 and \#2-\#3, respectively. In Figure 3 (c) we see two $20 \mathrm{GHz}$ waveforms that were programmed with a pi phase shift. The broad bandwidth and fine resolution, enabled by the 2D shaper geometry which accesses the huge number of pixels of the 2D LCOS display, made this experiment possible with plenty of bandwidth leftover, allowing for substantial future scalability to increase number of comb lines.

\section{References}

1. A. M. Weiner, Opt. Comm. 284, 3669-3692 (2011).

2. V. R. Supradeepa, C. B. Huang, D. E. Leaird, and A. M. Weiner, Opt. Exp. 16, 11878-11887 (2008).

3. V. R. Supradeepa, D.E.Leaird, and A.M. Weiner, International conf. Ultrafast phenomena, Snowmass, CO, 2010.

4. J. C. Vaughan, T. Hornung, T. Feurer, and K. A. Nelson, Opt. Lett. 30, 323-325 (2005).

5. Z. Jiang, C. B. Huang, D. E. Leaird, and A. M. Weiner, Nature Photon. 1, 463-467 (2007).

6. V. Torres-Company, A. J. Metcalf, D. E. Leaird, and A. M. Weiner, IEEE Photon. Technol. Lett. 24, 891-893 (2012). 